\title{
Railway Track Pedestrian Crossing between Platforms
}

\author{
G.Prabhavathi, B.Sanjana, Ms.S.P.Dhivya(Ap/Ece) \\ Department of Electronics and Communication Engg. SNS College of Engineering Coimbatore,India \\ Department of Electronics and Communication Engg. SNS College of Engineering Coimbatore,India
}

\begin{abstract}
This project is used for automatically close or open the mobile platforms in between the track trains. Normally the mobile platform connects the two platforms through which the passenger can walk on the platform to reach on the next platform Sensors are placed on the two sides of track. if the train reaches one sensor the mobile platform will automatically close and allows the train to go through the tracks and then when the train leaves the second sensor the mobile platform will automatically open the bridging platforms.The microcontroller will sense the presence of train by using infrared sensor. so on sensing the train on one path controller will give pulses to the stepper motor to close the mobile platform automatically.

Index Terms: IR-Sensor, Dc Gear Motor,Pic Microcontroller.
\end{abstract}

\section{INTRODUCTION}

The present railway systems in india are not automated which are fully manmade. In railway stations normally we use bridges.It is very difficult for the elderly persons or handicapped persons to use the bridge .This paper finds a good solution.Mainly the tracking of a train is sensed by sensor,this is used for automatically close/open the mobile platform. Sensors are placed on two sides of track to sense the motion of train.The microcontroller will sense the presence of trains by using infrared sensors. So on sensing the train on one path ,the controller will give pulses to the stepper motor to close the mobile platformautomatically.

\section{COMPONENT OF AUTOMATED SySTEM}

Embedded system is a system which comprises both hardware and software to perform specific function .It is design to do some specific task, rather than to be a general purpose computer for multiple task.The hardware components to be used are :

1.IR SENSOR:IR SENSOR is a device that emits and detects infrared radiation in order to sense some aspect of its surroundings.It can sense the heat of an object and detection motion. The radiation is invisible to our eyes but can be detected by an infrared sensor that accepts and interepts it.

2.LCD:It is a type of display used in digital watches and many portable computer .It utilize two sheets of polarizing material with a liquid solution between them .

3.LED:It is a semiconductor light source used as an indicator lamps and are used for lighting.

4.DC MOTOR:The gear having smaller radius will cover more RPM than the one with larger radius .The comparison between input and output gear gives the gear ratio.

\section{BLOCK DIAGRAM AND SPECIFICATION}

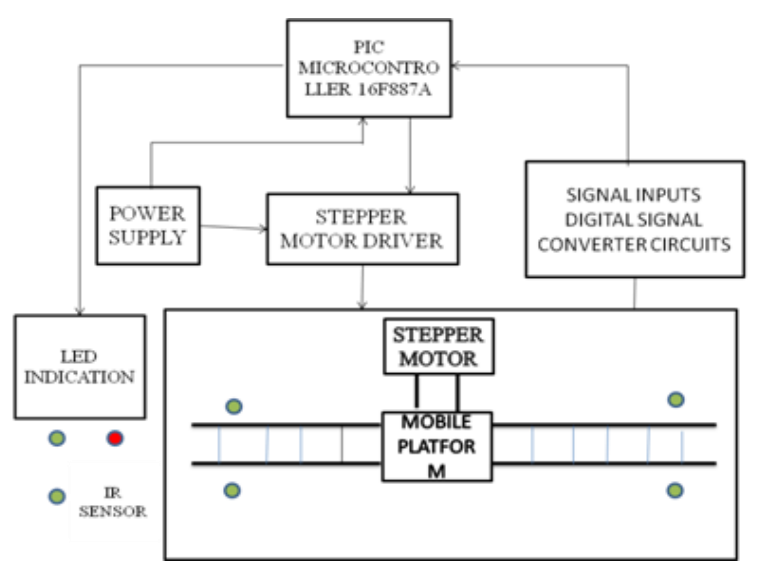

POWER SUPPLY:The main components used in power supply are transformer,rectifier,filter and regulator.The $230 \mathrm{~V}$ ac supply is converted into $12 \mathrm{~V}$ ac supply through the transformer.The output of the transformer has the same frequency as in the input ac power.The ac power is converted into dc power through the diodes.The bridge diode is used to convert the ac supply to dc supply.This converted dc supply has the ripple 
content and for the normal operation of the circuit,the ripple content of the dc power supply should be as low as possible because theripple content of the power supply will reduce the life of circuit.

1.TRANSFORMER: Transformers convert ac electricity from one voltage toanother voltage.Step-up transformers increase voltage, step-down transformer reduce voltage.Power supplies use a step-down transformer to reduce the high main voltage to a safer low voltage.

2.RECTIFIER: The rectifier is a device such as a semiconductor cableof converting sinusoidal input waveform units into a unidirectional waveform with a non zero average component.The input to the circuit is applied to the diagonially oppposite corners of the network and the output is takenfrom the remaining two corners.

3.FILTER: The capacitors are used as filters in the power supply unit.Shunting the load with the capacitor,effects filtering. The action of the system depends upon the fact the capacitor stores energy during the conduction period and delivers this energy to the load during the inverse or non conductng period.

4.REGULATOR:The voltage regulator comprise a class of widely used IC's.Regulator IC units contain the circuitry for reference source,comparator amplifier,control device,over load protection all in a single IC.IC units provide regulation of either a fixed positive voltage, a fixed negative voltage or an adjustable set voltage.

5.MICROCONTROLLER:Separate code and data spaces .A small number of fixed length instructions.Most instructions are single delay cycle upon branches and skips. The program isalso mapped into the data space and writable. It consist of a memory which is used to permanently save the program being executed.

6:DC MOTOR:The gear having smaller radius will cover more RPM than the one with larger radius.The larger gear will give more torque to the smaller gear than vice versa.Multiple gears are connected together,conservationof energy is followed.

7.LED:The wavelength of light emitted , and thus its color,depends on the band gap energy of the materials forming the $\mathrm{p}-\mathrm{n}$ junction.In silicon or germanium diodes, the electrons and holes recombine by anon-radiative transition, which produces no optical emission, because these are indirect band gapmaterials.

\section{INFRARED SENSOR}

An infrared sensor is an electronic device that emits and detects infrared radiation in order to sense somenaspect of its surrounding .It can measure the heat of an object, as well as detect motion.In a typical infrared sensor like a motion detector radiation enters the front an reaches the sensor itself at the center of the device.

WORKING PRINCIPLE OF IR

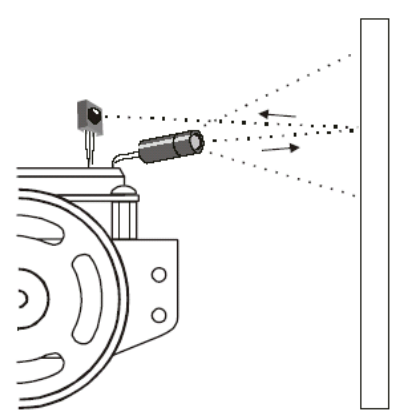

The IR detector is only looking for infrared that's flashing on and off 38500 times per second .It has built in optical filters that allow very little light except 980nm IR .It also has an electronic filter that only allows signals around $38.5 \mathrm{kHz}$ to pass through. When IR rays gets emitted from led, it moves in the direction it is angled. When any obstacle interferes in the path, the IR rays get cut and it produces secondary wavelets which propagates mostly in return direction or in a direction opposite to that of the primary waves, which produces the net result like reflection of IR rays .

\section{TYPES OF INFRARED SENSORS}

\section{- ACTIVE INFRARED SENSOR}

Active sensors employ both infrared source and infrared detectors .They operate by transmitting energy fron either a light emitting diode or laser diode .A led is used for a non-imaging active IR detector , and a laser diode is used for an imaging active IR detector.

\section{- BREAK BEAM SENSOR}

This type of sensor consist of a pair of light emitting and light detecting elements .Infrared source transmits a beam of light towards a remote IR receive creating an electronic fence. 


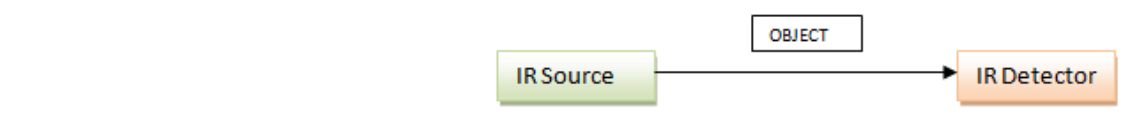

\section{- REFLECTANCE SENSOR}

This type of sensors house both an IR source and an IR detector in a single housing in such a way that light from emitter LED bounces off an external object and is reflected into a detector .Amount of light reflected into the detector depends upon the reflectivity of the surface.

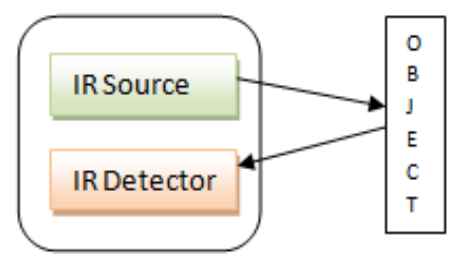

\section{Led And Its Specifications}

LEDs are used as indicator lamps in many devices and are increasingly used for lighting. When a light emitting diode is switched on ,electrons are able to recombine with holes within the device, releasing energy in the form of photons. This effect is called electroluminance, and the color of the light is determined by the energy band gap of the semiconductor.

WORKING PRINCIPAL OF LED

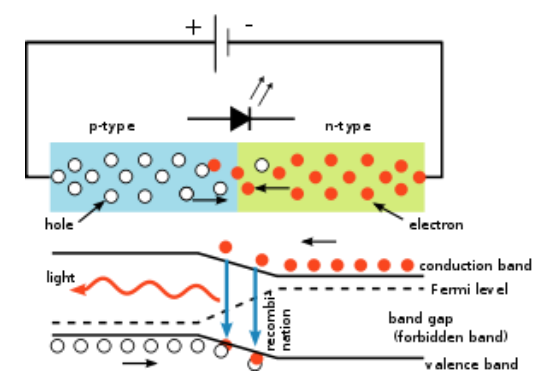

The LED consist of a chip of semiconducting material doped with impurities to creates p-n junction. Charge carriers electrons and holes flow into the junction from electrodes with different voltages. When an electron meets a hole, it falls into a lower energy level and releases energy in the form of a photon.

The of the light emitted, and thus its color,depends on the band gap energy of the material forming the pnjunction.The materials used for the LEDs have a direct band gap with energies corresponding to nearinfrared,visible, or near-ultraviolet light.

\section{COLORS AND MATERIALS}

\begin{tabular}{|l|l|l|}
\hline \multicolumn{1}{|c|}{ COLOR } & \multicolumn{1}{c|}{ WAVELENGTH } & \multicolumn{1}{c|}{ MATERIAL } \\
\hline Infrared & & Gallium arsenide \\
\hline Red & & Aluminium gallium \\
\hline Orange & & Indium phosphide \\
\hline Yellow & & Gallium phosphide \\
\hline
\end{tabular}

VI. Dc Gear Motor

DC motor works over a fair range of voltage .The higher the input voltage more is the RPM of the motor .if the motor works in the range of $6-12 \mathrm{~V}$, it will have the least RPM at $6 \mathrm{~V}$ and maximum at $12 \mathrm{~V}$.

$\mathrm{RPM}=\mathrm{K} 1 * \mathrm{~V}$, where,

$\mathrm{K} 1=$ induced voltage constant

$\mathrm{V}=$ voltage applied

\section{WORKING OF DC GEAR MOTOR}

The working of the gears is explained by the principal of conservation of angular momentum. The gear having smaller radius will cover more RPM than the one with larger radius, the larger gear will give more torque to the smaller gear than vice versa.RPM and torque are inversely proportional.The gear having more torque will provide a lesser RPM and converse . 


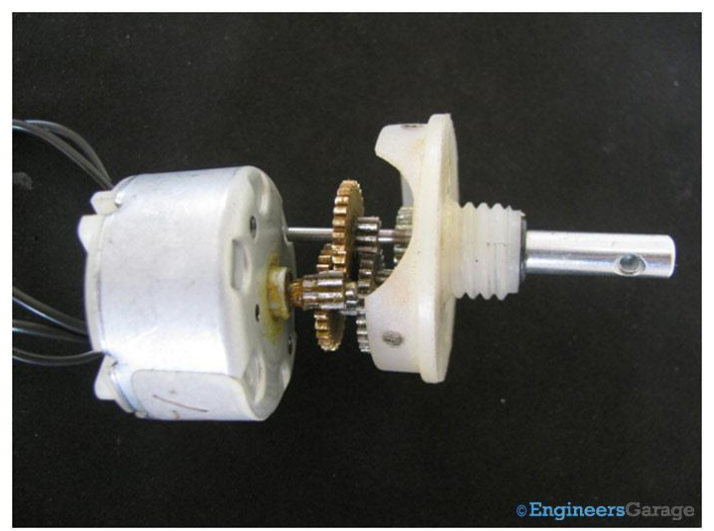

The gear connecting the motor and the gear head is quite small,it transfers more speed to the larger teeth part of the gear head and makes it rotate.The larger part of the gear further turns the smaller duplex part.The small duplex part receives the torque but not the speed from its predecessor which it transfers to larger part of other gear and so on.

VII. Relay Drive

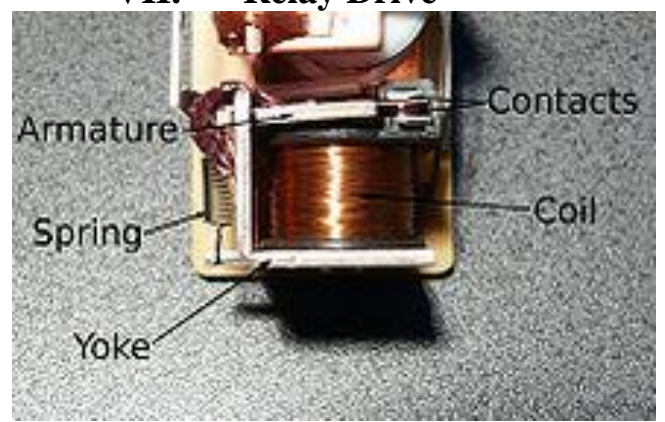

A relay is an electrically operated switch.Many relays use an electromagnet to operate a switching mechanism, but other operating principles are also used .Relays find application where it is necessary to control a circuit by a low-power signal.Relay found extensive use in telephones exchange and early computer to perform logical operations. Solid-state relays control power circuits with no moving parts,instead using a semiconductor device triggered by light to perform switching.

\section{WORKING PRINCIPLE}

A small electronic relay consist of a coil surrounding a soft iron core, an iron yoke, which produces a low reluctance path for magnetic flux,a movable iron armature, when the relay id de-energized there is an air-gap in the magnetic circuit. In this condition ,one of the two sets of contacts in the relay is closed and the other set is open.Other relays may have more or fewer sets of contacts depending on their function. When an electric current is passed through the coil, the resulting magnetic field attracts the armature, and the consequent moment of the movable contact or contacts either makes or breakes a connection with a fixed contact.If the set of contacts was closed when the relay was de-energized, then the movement opens the contacts and breaks the connection, if the contacts were open.

\section{RESULT}

This experiment is used for automatically close/open the mobile platform .It saves the time for passengers to cross the next platform. Thus the sensing is made continuously whenever the train arrives and pass through .

\section{CONCLUSION}

Thus the tracking of train is sensed continuously, which automatically close/open the mobile platform is partially automated which is beneficial for passengers to crossthe rail grade crossing. The system into a fully automated instead of climbing the staircase.This efficient method will be more compact for scheduling the train timings for reaching the particular destination and also for crossing the suitable platforms. 


\section{Reference}

[1]. U.S. Department of Transportation, Federal Highway Administration. Motor Vehicle Accident Costs (Technical Advisory T7570.2, 31 October).

[2]. Silla, A. and J. Luoma (2011). Effect of Three Counter measures against the Illegal Crossing of Railway Tracks. Accident Analysis and Prevention, Volume 43, Issue 3.

[3]. Delmonte, E. and S. Tong (2011). Improving Safety and Accessibility at Level 29 Crossings for Disabled Pedestrians. Report Number T650. Rail Safety and Standards 30 Board, London, United Kingdom, February 2011.

[4]. U.S. Department of Transportation, Federal Railroad Administration (2010). Railroad Trespassing, Vandalism, and Highway-Rail Grade Crossing Warning Device Violation Prevention Strategies. Office of Railroad Safety, December 2010.

[5]. Rail Safety and Standards Board (2010). Road-Rail Interface Special Topic Report. April 2010. London, United Kingdom

[6]. American Railway Engineering and Maintenance of Way Association (AREMA) (2010). Communications \& Signal Manual, 2010 Edition, Volume 1 .

[7]. 26. Korve, H.W., J.I. Farran, D.M. Mansel, H.S. Levinson, T. Chira-Chavala, and D.R. Ragland (1996). Integration of Light Rail Transit into City Streets. TCRP Report Transportation Research Board, Washington, D.C.

[8]. U.K. Department for Transport - Rail Accident Investigation Branch (2006). Rail Accident Report - Investigation into Station Pedestrian Crossings. Report 23/2006, December 2006.

[9]. Ogden, B.D. (2007). Railroad-Highway Grade Crossing Handbook - Revised Second 9 Edition 2007. Report No. FHWA-SA-07-010. Office of Safety Design Federal 10 Highway Administration. 